\title{
Diagnostic Accuracy of Chest Ultrasound versus Plain Chest X-ray in Acute Assessment of Traumatic Hemothorax Samir Mohamed Attia ${ }^{1}$, Noureldin Noaman Gwely ${ }^{2}$, Mohamed El-Said Ibrahim ${ }^{3}$, Mahitab Galal El-Din Rashwan Hefny*4 Departments of ${ }^{1}$ Vascular Surgery, ${ }^{2}$ Cardiothoracic Surgery, ${ }^{3}$ Critical Care Medicine and ${ }^{4}$ Emergency Medicine - Faculty of Medicine - Mansoura University, Egypt *Corresponding author: Mahitab Galal El-Din Rashwan Hefny, Mobile: (+20) 01114496850, E-Mail: mahimosalam@yahoo.com
}

\section{ABSTRACT}

Background: Trauma is the leading cause of death in young adults, as well as a significant cause of morbidity and mortality in people of all ages. Ultrasonography (US) is a good first imaging modality because it is fast, repeatable, and noninvasive. It can also be achieved at the same time as other resuscitative steps, providing critical information without the time delay caused by radiograph or CT scan execution and interpretation.

Objective: To compare the diagnostic accuracy of chest ultrasound with plain chest X-ray in acute assessment of traumatic hemothorax at Emergency Hospital, Mansoura University.

Patients and methods: This was a cross-sectional study included a total of 109 patients with polytrauma and conducted at Emergency Medicine Department, Mansoura University from November 2019 to October 2020. Emergency Hospital, Mansoura University is a level one trauma center with about 250,000 visit and 25,000 trauma cases per year. Results: The X-ray findings in the cases included in the study where fracture ribs was detected in 76 cases (69.7\%), lung contusions in 28 cases $(25.7 \%)$, obliteration of costophrenic angles in 17 cases (15.6\%) and tension pneumothorax in 38 cases (34.9\%). US showed $86.2 \%$ sensitivity, $100 \%$ specificity, PPV 100\%, NPV 95.2\% and $96.3 \%$ accuracy. X-ray showed $58.6 \%$ sensitivity, $100 \%$ specificity, PPV $100 \%$, NPV $86.9 \%$ and $89 \%$ accuracy. Conclusion: Ultrasound has significant value in initial assessment of life-threatening conditions of trauma with acceptable sensitivity and high specificity.

Keywords: Chest Ultrasound, Plain Chest X-ray, Traumatic Hemothorax.

\section{INTRODUCTION}

Hemothorax refers to a collection of blood within the pleural cavity. By definition this bloody pleural effusion should contain a hematocrit value of at least $50 \%$ of the hematocrit of peripheral blood ${ }^{(1)}$. The primary cause of hemothorax is sharp or blunt trauma to the chest. Iatrogenous and spontaneous hemothoraces occur less frequently. Thoracic trauma continues to be a substantial cause of morbidity and mortality. Chest injuries occur in approximately $60 \%$ of multitrauma patients and are responsible for $20 \%$ to $25 \%$ of trauma-related mortalities ${ }^{(2)}$.

Although some thoracic traumas are treated according to clinical findings of the patient before performing any imaging studies, but in many cases application of various imaging modalities such as computed tomography (CT) scan, plain chest X-ray (CXR) and ultrasonography are necessary. Among these modalities, CT scan is the gold standard for identification of intrathoracic injuries following trauma with a significantly high diagnostic value for occult and soft tissue injuries. However, limited availability of CT scan in all medical centers, overcrowding of the emergency department and radiation exposure led the researchers to look for other diagnostic tools ${ }^{(3)}$.

CXR is the first diagnostic test for screening of thoracic traumas but the limitations of supine radiography in some traumatic injuries is confirmed in various studies. Moreover, Low diagnostic yield of routine chest radiography in patients with thoracic injuries encouraged the researchers to search for alternative imaging techniques ${ }^{(4)}$.

The use of ultrasonography became a mainstay in emergency department and trauma evaluation. Ultrasonography is often more readily attainable than CT and can be used in patients who are not stable enough for transport ${ }^{(5)}$. Major attention has recently been drawn to ultrasonography as a quick screening tool with minimum complications. It has shown to have superior diagnostic value in detection of thoracic traumatic injuries. However, diagnostic accuracy of ultrasonography is highly dependent on the skills of the operator and is usually not reliable in detection of injuries without bleeding or free fluid ${ }^{(6)}$.

Many reports point out the advantage of ultrasonography in the detection, diagnosis, follow-up and planning initial therapy of the hemothorax in major trauma patients. Percutaneous diagnostic interventions under US are widely accepted as useful procedure with high diagnostic efficacy and low complication rate ${ }^{(7)}$.

The aim of this study was to compare the diagnostic accuracy of chest ultrasound with plain chest X-ray in acute assessment of traumatic hemothorax at Emergency Hospital, Mansoura University.

\section{PATIENTS AND METHODS}

This was a cross-sectional study a total of 109 patients with polytrauma conducted at Emergency Medicine Department, Mansoura University from November 2019 to October 2020. Emergency Hospital 
Mansoura University is a level one trauma center with about 250,000 visit and 25,000 trauma cases per year. Inclusion criteria: Patients of all ages and genders that have suffered chest trauma, whether blunt, penetrating, or iatrogenic.

Exclusion criteria: Hemodynamic instability, old trauma more than 24 hours, patients in need for emergent procedures or transport to the operating room, pregnancy and patients who do not wish to participate in the study.

\section{Methods:}

1) Clinical evaluation:

- Demographic data; age, gender, and mechanism of trauma.

- Clinical examination of the patients at the Trauma Room including vital signs, Glasgow coma scale, complete general examination.

- Advanced Trauma Life Support ${ }^{\circledR}$ (ATLS $\left.{ }^{\circledR}\right)^{(8)}$.

\section{2) Laboratory work up:}

- $\mathrm{CBC}$.

- Liver function tests.

- Serum creatinine.

- Coagulation profile (PT, INR, and APTT).

- ABO grouping.

- ABG.

\section{3) Radiological work up:}

- FAST (Focused assessment sonography for trauma patient).

- Plain chest X-ray (an anterior posterior (AP) chest $\mathrm{X}$-ray, supine films using a portable $\mathrm{X}$-ray machine )

- Chest ultrasound.

- CT chest.

Ethical consideration:

An approval of the study was obtained from Mansoura University academic and ethical committee. Informed written consent was obtained from the relatives of the patients sharing in the study. Confidentiality and personal privacy were respected in all levels of the study. Collected data will not be used for any othr purpose.

\section{Statistical analysis}

The collected data were coded, processed and analyzed using the SPSS (Statistical Package for the Social Sciences) version 22 for Windows ${ }^{\circledR}$ (IBM SPSS Inc, Chicago, IL, USA). Qualitative data were represented as frequencies and relative percentages. Fisher's exact test $\left(\chi^{2}\right)$ was used to compare qualitative variables. Quantitative data were expressed as mean \pm $\mathrm{SD}$ (Standard deviation), median, and range. $\mathrm{P}$ value $<0.05$ was considered significant.

\section{RESULTS}

Table 1 shows the demographic data of cases included in study. Blunt trauma was the most common type of trauma. Right side of the chest was affected in 41 cases, left side in 36 and there was bilateral affection in 32 cases.

Table (1): Demographic data, mode of trauma and site of trauma in the cases included in the study

\begin{tabular}{|c|c|c|}
\hline \multicolumn{2}{|l|}{ Items } & Study cases $(n=109)$ \\
\hline \multirow{2}{*}{$\begin{array}{l}\text { Age } \\
\text { (years) }\end{array}$} & \begin{tabular}{|l} 
Mean \pm \\
SD
\end{tabular} & $34.57 \pm 9.67$ \\
\hline & \begin{tabular}{|l}
$\begin{array}{l}\text { Median } \\
(\text { min-max) }\end{array}$ \\
\end{tabular} & $36(18-63)$ \\
\hline \multicolumn{3}{|l|}{ Sex } \\
\hline \multicolumn{2}{|c|}{ Females } & $25(22.9 \%)$ \\
\hline Males & & $84(77.1 \%)$ \\
\hline \multicolumn{3}{|c|}{ Mode of trauma (MOT): } \\
\hline \multicolumn{2}{|c|}{ Blunt trauma } & $87(79.8 \%)$ \\
\hline \multicolumn{2}{|c|}{ Penetrating trauma } & $22(20.2 \%)$ \\
\hline \multicolumn{2}{|c|}{-Fire arm injuries } & $7(6.4 \%)$ \\
\hline \multicolumn{2}{|c|}{$\begin{array}{l}\text {-Sharp weapons } \\
\text { (including stab) }\end{array}$} & $15(13.8 \%)$ \\
\hline \multicolumn{3}{|c|}{ Site of trauma: } \\
\hline \multicolumn{2}{|c|}{ Right side } & $41(37.6 \%)$ \\
\hline \multicolumn{2}{|c|}{ Left side } & $36(33 \%)$ \\
\hline \multicolumn{2}{|c|}{ Bilateral } & $32(29.4 \%)$ \\
\hline
\end{tabular}

Table (2) shows the X-ray, ultrasound (US), and CT findings in the cases included in the study.

Table (2): Analysis of the X-ray, US and CT findings in the cases included in the study

\begin{tabular}{|l|c|}
\hline Items & Study cases (n= 109) \\
\hline X-ray findings & $\mathbf{7 6}(\mathbf{6 9 . 7 \%})$ \\
\hline Fracture ribs & $\mathbf{2 8}(\mathbf{2 5 . 7 \%})$ \\
\hline lung contusions & $\mathbf{1 7}(\mathbf{1 5 . 6 \%})$ \\
\hline $\begin{array}{l}\text { Obliteration of } \\
\text { costophrenic angles }\end{array}$ & $\mathbf{3 8}(\mathbf{3 4 . 9 \%})$ \\
\hline Tension pneumothorax & $\mathbf{2 5 ( 2 2 . 9 \% )}$ \\
\hline US findings: & $\mathbf{8 4}(\mathbf{7 7 . 1 \%})$ \\
\hline $\begin{array}{l}\text { Obliteration of } \\
\text { costophrenic angle }\end{array}$ & $\mathbf{7 4 ( 6 7 . 9 \% )}$ \\
\hline $\begin{array}{l}\text { No Obliteration of } \\
\text { costophrenic angle }\end{array}$ & $\mathbf{6 ( 5 . 5 \% )}$ \\
\hline CT findings: & $\mathbf{3 5 ( 3 2 . 1 \% )}$ \\
\hline Fracture ribs & $\mathbf{2 5 ( 2 2 . 9 \% )}$ \\
\hline Fracture sternum & $\mathbf{4 ( 3 . 7 \% )}$ \\
\hline Lug contusions & $\mathbf{4 0 ( 3 6 . 7 \% )}$ \\
\hline Hemothorax & $\mathbf{1 4 ( 1 2 . 8 \% )}$ \\
\hline Hemopneumothorax
\end{tabular}

As regard the association between CT and US in detection of hemothorax, US showed negative hemothorax in 84 cases, among them 80 cases were proved to be negative by $\mathrm{CT}$ while 4 cases were positive by CT (Table 3 ). 
Table (3): Correlation between US and CT in detection of hemothorax

\begin{tabular}{|c|c|c|c|c|c|}
\hline & \multicolumn{4}{|c|}{$\overline{\text { CT findings }}$} & \multirow{3}{*}{$\mathbf{P}$} \\
\hline & \multicolumn{2}{|c|}{$\begin{array}{c}\text { Negative } \\
(n=80)\end{array}$} & \multicolumn{2}{|c|}{$\begin{array}{c}\text { Positive } \\
(n=29)\end{array}$} & \\
\hline & No & $\%$ & No & $\%$ & \\
\hline US findings & & & & & \\
\hline Negative $(\mathrm{N}=84)$ & 80 & 100 & 4 & 13.8 & $<0001^{*}$ \\
\hline Positive $(\mathrm{N}=25)$ & 0 & 0 & 25 & 86.2 & \\
\hline
\end{tabular}

*: Statistically significant

As regard the association between $\mathrm{CT}$ and X-ray in detection of hemothorax, X-ray showed negative hemothorax in 92 cases, among them 80 cases were proved to be negative by CT while 12 cases were positive by CT (Table 4).

Table (4): Correlation between X-ray and CT in detection of hemothorax

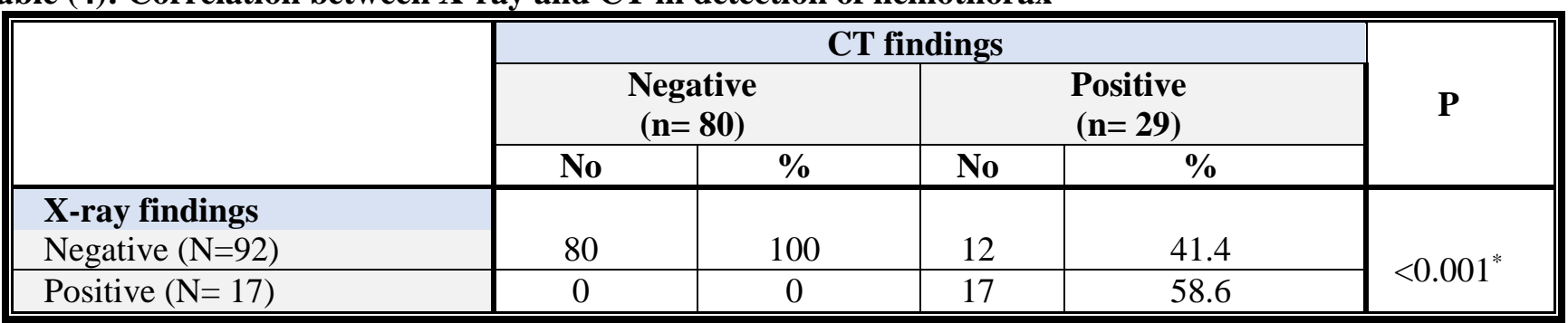

*: Statistically significant

As compared with CT findings, the diagnostic test accuracy of US and X-ray is shown in Table 5.

Table (5): Diagnostic accuracy of US versus X-ray in detection of hemothorax as compared with CT

\begin{tabular}{|c|c|c|c|c|}
\hline & \multicolumn{4}{|c|}{ CT findings } \\
\hline & \multicolumn{2}{|c|}{$\begin{array}{c}\text { Negative } \\
(n=80)\end{array}$} & \multicolumn{2}{|c|}{$\begin{array}{c}\text { Positive } \\
(n=29)\end{array}$} \\
\hline & No & No & No & No \\
\hline \multicolumn{5}{|l|}{ X-ray findings } \\
\hline Negative $(\mathrm{N}=92)$ & $80(\mathrm{TN})$ & 100 & $12(\mathrm{FN})$ & 41.4 \\
\hline Positive $(\mathrm{N}=17)$ & $0(\mathrm{FP})$ & 0 & 17 (TP) & 58.6 \\
\hline Sensitivity & \multicolumn{4}{|c|}{$58.6 \%$} \\
\hline Specificity & \multicolumn{4}{|c|}{$100 \%$} \\
\hline PPV & \multicolumn{4}{|c|}{$100 \%$} \\
\hline NPV & \multicolumn{4}{|c|}{$86.9 \%$} \\
\hline Accuracy & \multicolumn{4}{|c|}{$89 \%$} \\
\hline \multicolumn{5}{|l|}{ US findings } \\
\hline Negative $(\mathrm{N}=84)$ & $80(\mathrm{TN})$ & 100 & $4(\mathrm{FN})$ & 13.8 \\
\hline Positive $(\mathrm{N}=25)$ & 0 (FP) & 0 & $25(\mathrm{TP})$ & 86.2 \\
\hline Sensitivity & \multicolumn{4}{|c|}{$86.2 \%$} \\
\hline Specificity & \multicolumn{4}{|c|}{$100 \%$} \\
\hline PPV & \multicolumn{4}{|c|}{$100 \%$} \\
\hline NPV & \multicolumn{4}{|c|}{$95.2 \%$} \\
\hline Accuracy & \multicolumn{4}{|c|}{$96.3 \%$} \\
\hline
\end{tabular}




\section{DISCUSSION}

The study included 135 cases who were eligible for enrollment in the study. Among the cases, 11 cases arrested on ED, 10 cases required urgent surgical interference and 5 cases refused to participate. The final number included was 109 cases.

In this study, the mean age of the cases was 34.57 , the minimum age was 18 years and the maximum age was 63 years. $77.1 \%$ of the cases were males. Blunt trauma was the most common type of trauma (79.8\%). This agreed with Salama et al. ${ }^{(\mathbf{9})}$ who showed that males were more common than females (92\%), the mean age was 32 years old, and blunt trauma (due to motor car accident and falling from height) was more than penetrating one (80\%). Our results also came in accordance with Vargas et al. ${ }^{(10)}$ who showed that among seventy-six patients included in their study, there were 60 males (79\%). The age range was between 18 and 82 years, with a mean age of 32 years, and median (interquartile rang) of 32.5 (25-50). Closed chest injury was the most common type of trauma (77\%). The results of the current study also came in agreement with Ianniello et al. ${ }^{(11)}$ who included 3320 consecutive major traumatic patients who were admitted to their Emergency Department (1844 men and 1476 women, with a median age of 41 years and an age range of 18-81 years). Of these patients, 3088 suffered a blunt trauma, and 232 a penetrating trauma.

The blunt chest trauma was the most prevalent type of trauma, which was in agreement with most of the studies. However, in the study conducted by Awais et al. ${ }^{(12)}$ penetrating and blunt thoracic trauma was reported in $104(50.2 \%)$ and $103(49.8 \%)$ cases respectively with nearly equal distribution. Furthermore, Khorsandi et al. ${ }^{(13)}$ study showed more prevalence in penetrating trauma patients than those with blunt trauma among studied patients.

In the current study, the right side of the chest was affected in $37.6 \%$, left side in $33 \%$ and there was bilateral affection in $29.4 \%$ of cases. The affected side showed variation between the different studies with no predominance of a specific side in affection by trauma. For example, Hwang and Lee ${ }^{(14)}$ in their study showed that among 201 patients with chest trauma included in their study, locations of injury included left 95 cases, right 67 cases, both 12 cases, and sternum 26 cases.

In the current study, among the included cases, there was 13 asymptomatic cases (11.9\%) and 96 symptomatic cases $(88.1 \%)$. The main symptoms included chest pain (79.2\%), palpitation (32.3\%), cyanosis $(16.5 \%)$ and orthopnea $(12.5 \%)$. The signs included tenderness $(82.3 \%)$, hypoxia $(25 \%)$, shock $(38.5 \%)$, tachypnea $(46.9 \%)$ and tachycardia $(35.8 \%)$. Similar results were reported by Salama et al. ${ }^{(9)}$ who showed that the most common presentation of the patients with chest trauma is chest pain (88\%), and dyspnea $(72 \%)$.

In the current study, the X-ray findings in the cases included in the study showed that fracture ribs was detected in $69.7 \%$ while by CT findings, fracture ribs was in $67.9 \%$. Our results are in concordance with previous studies, which have also reported a slightly higher prevalence of rib fractures among patients with blunt trauma as compared to those with penetrating trauma (15, 16). Yet, much lower incidence of rib fractures was reported by Awais et al. ${ }^{(12)}$ who showed that the overall prevalence of rib fractures in their study sample was $36.2 \%$. Among patients with blunt and penetrating trauma, the prevalence of rib fractures was $39.8 \%$ and $32.7 \%$ respectively.

In this study, lung contusions were in $25.7 \%$ of cases as detected by $\mathrm{x}$-ray while lug contusions were in $32.1 \%$ as detected by CT. According to Rashid $\boldsymbol{e t}$ al. (17) pulmonary contusion is considered to be a relatively benign lesion that does not add to the morbidity or mortality of patients with blunt chest trauma, if managed properly. According to Huber et al. ${ }^{(18)}$ lung injuries such as contusions or lacerations were only predictive of mortality if major or bilateral.

CT findings in the cases included in the study showed fracture ribs in $67.9 \%$, fracture sternum in $5.5 \%$, lung contusions in $32.1 \%$, hemothorax in $22.9 \%$, hemopneumothorax in $3.7 \%$, tension pneumothorax in $36.7 \%$ and mediastinal injury in $12.8 \%$. In the study conducted by Vafaei $\boldsymbol{e t}$ al. ${ }^{\left({ }^{(19)}\right)}$ that included 152 chest trauma patients, chest CT scan showed pulmonary contusion in $48(31.6 \%)$ patients, hemothorax in 29 $(19.1 \%)$, and pneumothorax in $55(36.2 \%)$ cases. Similar results were shown by Vargas et al. ${ }^{(10)}$ who showed that there were 13 patients with hemothorax in the right lung and 20 with hemothorax in the left lung as diagnosed with chest CT; while 11 patients with hemothorax in the right lung and 15 with hemothorax in the left lung were diagnosed via sonographic study (10).

In the current study, as compared with CT findings, X-ray showed $58.6 \%$ sensitivity, $100 \%$ specificity, PPV 100\%, NPV $86.9 \%$ and $89 \%$ accuracy. As compared with CT findings, US showed $86.2 \%$ sensitivity, $100 \%$ specificity, PPV 100\%, NPV $95.2 \%$ and $96.3 \%$ accuracy. The diagnostic accuracy of US was higher as compared with X-ray. This came in accordance with Vafaei et al. ${ }^{(19)}$ who showed higher accuracy of US in detection of hemothorax following chest trauma. Area under the ROC curve of ultrasonography in detection of hemothorax, was 0.86 (95\% CI: 0.78-0.94). Area under the ROC curve of $\mathrm{x}-$ ray was 0.77 (95\% CI: $0.68-0.86)$ for hemothorax. However, the diagnostic value of the two tests was equal in detection of hemothorax $(\mathrm{p}=0.08)$. Hyacinthe et al. (3) showed that the diagnostic accuracy of ultrasonography was higher than that of chest x-ray. The study showed that the sensitivity and specificity of ultrasonography, compared to CT scan as the gold standard, in diagnosis of thoracic cavity lesions were in the $37-61 \%$ and $61-96 \%$ ranges, respectively. The sensitivity and specificity of US in detection of hemothorax in the study conducted by Vargas $\boldsymbol{e t}$ al. ${ }^{(\mathbf{1 0})}$ were $78.7 \%$ and $92.6 \%$, respectively, with a positive predictive value and negative predictive value of $65 \%$ 
and $97.8 \%$, respectively as compared with CT. Wilkerson and Stone meta-analysis reported a sensitivity of $85-100 \%$ for ultrasonography in diagnosis of thoracic cavity injuries ${ }^{(20)}$. Other studies, have also reported similar findings ${ }^{(21,22)}$.

In the emergency room, hemothorax is usually diagnosed by physical examination and chest $\mathrm{x}$-ray. Although physical examination in the context of trauma in the emergency room has limited sensitivity and specificity ${ }^{(23)}$.

\section{CONCLUSION}

- Ultrasound has significant value in initial assessment of life threatening conditions of trauma with acceptable sensitivity and high specificity.

- Ultrasound has the benefits of being cheap, compact, and readily available, as well as being able to conduct multi-planar imaging and releasing no ionizing radiation.

- Chest ultrasound has a high degree of sensitivity when it comes to detecting multiple chest injuries.

- Ultrasonography has a greater sensitivity than radiography when it comes to detecting a hemothorax.

- Emergency chest ultrasound, conducted as part of the primary survey of the traumatized patient, can diagnose hemothorax quickly and reliably, and is a useful method to supplement the immediate clinical assessment of these patients.

\section{REFERENCES}

1. Boersma W, Stigt J, Smit H (2010): Treatment of haemothorax. Respir Med., 104(11): 1583-1587.

2. Broderick S (2013): Etiology, diagnosis, and management. Thorac Surg Clin., 23: 89-96.

3. Hyacinthe A, Broux C, Francony G et al. (2012): Diagnostic accuracy of ultrasonography in the acute assessment of common thoracic lesions after trauma. Chest, 141(5): 1177-1183.

4. Forouzanfar M, Safari S, Niazazari M et al. (2014): Clinical decision rule to prevent unnecessary chest X-ray in patients with blunt multiple traumas. Emerg Med Aust., 26(6): 561-566.

5. Brooks A, Davies B, Smethhurst M et al. (2004): Emergency ultrasound in the acute assessment of haemothorax. Emergency Medicine Journal, 21(1): 44-46.

6. Michalke J (2012): An overview of emergency ultrasound in the United States. World J Emerg Med., 3(2): 85-90. 3(2): 85 .

7. McEwan K, Thompson P (2007): Ultrasound to detect haemothorax after chest injury. Emerg Med J., 24(8): 581582.

8. Sumann G, Moens D, Brink B et al. (2020): Multiple trauma management in mountain environments - a scoping review. Scandinavian Journal of Trauma, Resuscitation and Emergency Medicine, 28: 117.
9. Salama K, Elshaboury I, Huissen W et al. (2017): Role of bedside sonography in the assessment of patients with chest trauma in the emergency department of Suez Canal University Hospital. International Surgery Journal, 4(2): 465-471.

10. Vargas C, Quintero J, Figueroa $\mathrm{R}$ et al. (2020): Extension of the thoracic spine sign as a diagnostic marker for thoracic trauma. European Journal of Trauma and Emergency Surgery, 6: 1-7.

11.Ianniello S, Piccolo L, Trinci M et al. (2019): ExtendedFAST plus MDCT in pneumothorax diagnosis of major trauma: time to revisit ATLS imaging approach? Journal of Ultrasound, 22(4): 461-469.

12. Awais M, Salam B, Nadeem N et al. (2019): Diagnostic accuracy of computed tomography scout film and chest Xray for detection of rib fractures in patients with chest trauma: a cross-sectional study. Cureus, 11(1): (1):3875-9.

13. Khorsandi M, Skouras C, Prasad S et al. (2015): Major cardiothoracic trauma: Eleven-year review of outcomes in the North West of England. The Annals of The Royal College of Surgeons of England, 97(4): 298-303.

14. Hwang E, Lee $Y$ (2016): Simple $X$-ray versus ultrasonography examination in blunt chest trauma: effective tools of accurate diagnosis and considerations for rib fractures. Journal of Exercise Rehabilitation, 12(6): 637-42.

15.Farooq U, Raza W, Zia N et al. (2006): Classification and management of chest trauma. Journal-College of Physicians and Surgeons of Pakistan, 16(2): 101-106.

16.Parvez M, Malik U, Ali M et al. (2007): Management of thoracic injuries: Our experience at military hospitals. Pakistan Armed Forces Medical Journal, 57(2): 104-112.

17. Rashid M, Wikström T, Örtenwall P (2000): Outcome of lung trauma. European Journal of Surgery, 166(1): 2228.

18. Huber S, Biberthaler P, Delhey $P$ et al. (2014): Predictors of poor outcomes after significant chest trauma in multiply injured patients: a retrospective analysis from the German Trauma Registry (Trauma Register DGU®): Scandinavian. Journal of Trauma, Resuscitation and Emergency Medicine, 22(1): 52-7.

19. Vafaei A, Hatamabadi H, Heidary $K$ et al. (2016): Diagnostic accuracy of ultrasonography and radiography in initial evaluation of chest trauma patients. Emergency, 4(1): 29-35.

20.Wilkerson G, Stone M (2010): Sensitivity of bedside ultrasound and supine anteroposterior chest radiographs for the identification of pneumothorax after blunt trauma. Academic Emergency Medicine, 17(1): 11-17.

21. Soldati G, Testa A, Sher S et al. (2008): Occult traumatic pneumothorax: diagnostic accuracy of lung ultrasonography in the emergency department. Chest, 133(1): 204-211.

22. Roberts D, Niven D, James M et al. (2014): Thoracic ultrasonography versus chest radiography for detection of pneumothoraces: challenges in deriving and interpreting summary diagnostic accuracy estimates. Critical Care, 18(2): 1-3.

23. Atkinson P, Milne J, Loubani $O$ et al. (2012): The Vline: a sonographic aid for the confirmation of pleural fluid. Critical Ultrasound Journal, 4(1): 19-23. 